\title{
Exact analytical solutions for some popular benchmark problems in topology optimization III: L-shaped domains revisited
}

\author{
T. Lewiński • G. I. N. Rozvany • \\ T. Sokót $\cdot$ K. Bołbotowski
}

Received: 8 June 2012 / Revised: 7 September 2012 / Accepted: 9 November 2012 / Published online: 3 January 2013

(C) The Author(s) 2012. This article is published with open access at Springerlink.com

\begin{abstract}
The paper provides the corrections and extensions of the paper of the same title, published in vol. 35, 2008 , pp. 165-174 of the current journal. The analytical solutions of Michell trusses constructed within the L-shaped domains are confirmed by the ground structure method of the truss optimization, thus giving the upper bounds of the exact solutions.
\end{abstract}

Keywords Michell trusses - Minimum weight design .

Topology optimization · Ground structure

\section{Introduction}

The recently published software aimed at predicting the optimal layouts of trusses of minimal weight, see Sokół (2011a, b), originally based on the truss ground structure filling up a fixed rectangular domain, has been effectively extended to other shapes of feasible domains, including the

T. Lewiński · T. Sokół $(\bowtie) \cdot$ K. Bołbotowski

Department of Structural Mechanics and Computer Aided

Engineering, Institute of Building Engineering, Faculty of Civil

Engineering, Warsaw University of Technology,

Al. Armii Ludowej 16, 00-637 Warsaw, Poland

e-mail: t.sokol@il.pw.edu.pl

T. Lewiński

e-mail: t.lewinski@il.pw.edu.pl

K. Bołbotowski

e-mail: k.bolbotowski@il.pw.edu.pl

\section{G. I. N. Rozvany}

Department of Structural Mechanics,

Budapest University of Technology and Economics,

Müegyetem rkpt. 3, Kmf. 35, 1521, Budapest, Hungary

e-mail: smo.rozvany@t-online.hu domains of polygons, allowing also for non-convex shapes. The current version of the program developed makes use of the adaptive ground structure approach with the selective subsets of active bars (Sokół 2011b). This approach is similar in spirit to that proposed by Gilbert and Tyas (2003) but applies a different strategy of adding-removing of the active bars in the fully connected ground structure. The program is capable of solving large-scale problems with the number of potential members exceeding one billion $\left(10^{9}\right)$ which provides highly accurate results both for volume and layout of fully stressed truss of minimal weight, designed within a given feasible domain, supported along given parts of the boundary or at fixed supports and subjected to a loading applied at fixed points. In the present paper we shall confine attention to the case of the absolute value of the lower and upper limits for the stress being equal, denoted by $\sigma_{0}$. Equipped with this new software one can solve numerically a broad class of the optimum design problems of Michell type for the L-shaped domains thus checking the analytical solutions predicted by Lewiński and Rozvany (2008). The noted discrepancies between the numerical predictions and the analytical results mentioned were the impulse for the present authors to look at this class of problems more closely. The result of the current verification is the confirmation of all the layouts predicted in Lewiński and Rozvany (2008) in the case of the point load being directed vertically (or parallel to the vertical edges of the L-shaped domain). However, the formulae for the optimal weight should be corrected. The source of the mistake was neglecting the rigid rotation $\omega$ at the vicinity of the re-entrant corner. The present paper makes up for this shortcoming, by deriving the new and now fully correct formulae for the virtual (adjoint) displacement fields within the feasible L-shaped domains, leading to analytical formulae for the optimum weight. The new formulae provide the results which 
compare favorably with the numerical predictions involving up to $10^{9}$ truss members.

The cases of the force being non-vertical need a special care. The numerical predictions reveal new, till now never published layouts. Some of them lie within the scope of the formulae derived for the case of the vertical forces, while other are completely different, hence their analytical discussion is planned to be the subject of a future research.

For brevity, Eq.n, Fig.m and Sec.p of Lewiński and Rozvany (2007) (or Lewiński and Rozvany 2008) will be referred to as Eq.II.n, Fig.II.m and Sec.II.p (or Eq.III.n, Fig.III.m and Sec.III.p). The notation used is adopted after the papers referred. The present paper can be viewed as the addendum and extension of the publication by Lewiński and Rozvany (2008).

\section{Optimal topology for an inclined line support and point load}

We revisit the Section III.2. Note that Eq.II.78 refers to the circular domain in Fig.III.3; hence the rigid rotation is expressed by: $\omega(\alpha, \beta)=-2 \beta+d_{1}$, which corrects the formula for $\omega$ given in Sec.III.2 (below Fig.III.3). In fact, the quantity $\omega(0,0)$, or the rigid rotation measured at the corner B, see Fig.III.3, is equal to a constant $d_{1}$ and does not vanish, as assumed in the paper revisited. This shortcoming will change the results concerning the adjoint displacement fields in the domain adjacent to the circular fan.

The Cartesian coordinates of points $(x, y)$ in the domain $\mathrm{BPG}_{1}$, see Fig.III.3, are given by

$$
\begin{aligned}
& x(\alpha, \beta) / a=\bar{x}(\alpha, \beta) \cos (\beta-\alpha)-\bar{y}(\alpha, \beta) \sin (\beta-\alpha), \\
& y(\alpha, \beta) / a=\bar{x}(\alpha, \beta) \sin (\beta-\alpha)+\bar{y}(\alpha, \beta) \cos (\beta-\alpha),
\end{aligned}
$$

where $\bar{x}(\alpha, \beta), \bar{y}(\alpha, \beta)$ are given by Eqs.III.3. Thus the position of point $\mathrm{P}$ with coordinates $\left(\alpha_{p}, \beta_{p}\right)$ is determined by

$a_{1}=y\left(\alpha_{p}, \beta_{p}\right), \quad b_{p}=-x\left(\alpha_{p}, \beta_{p}\right)$,

see Figs.III.2, III.3. Note that in Fig.III.3 we have: $\beta_{p}=\theta$. The incorrect assumption $\omega(0,0)=0$ made in Sec.III.2, is corrected here, thus leading to the following new form of equations III.10:

$$
\begin{gathered}
u(\xi, \eta)=\bar{u}_{0}(\xi, \eta)+\int_{0}^{\xi}\left[\cos (\xi-\alpha)+\left(\omega_{0}+2 \eta+2 \alpha\right)\right. \\
\quad \times \sin (\xi-\alpha)] A(\alpha, \eta) d \alpha
\end{gathered}
$$

$$
\begin{gathered}
v(\xi, \eta)=\bar{v}_{0}(\xi, \eta)+\int_{0}^{\xi}\left[\sin (\xi-\alpha)-\left(\omega_{0}+2 \eta+2 \alpha\right)\right. \\
\quad \times \cos (\xi-\alpha)] A(\alpha, \eta) d \alpha
\end{gathered}
$$

with $\omega_{0}=-\omega(0,0)$ or $\omega_{0}=-d_{1}$ and since $d_{1}=-1-\pi$, see the result below Eqs III.6, we have

$\omega_{0}=1+\pi$,

while $\bar{u}_{0}, \bar{v}_{0}$ are defined by Eq.III.11. The results III.12, III.13 should be corrected to the form

$$
\begin{aligned}
u(\xi, \eta) / a= & (1+k)\left[F_{1}(\xi, \eta)-F_{3}(\xi, \eta)\right] \\
& +2(\eta-\xi) G_{1}(\xi, \eta)+G_{0}(\xi, \eta)+2 F_{2}(\xi, \eta), \\
v(\xi, \eta) / a= & (1+k)\left[F_{2}(\xi, \eta)-F_{0}(\xi, \eta)\right] \\
& -2(\eta-\xi) G_{0}(\xi, \eta)+G_{1}(\xi, \eta)-2 F_{1}(\xi, \eta),
\end{aligned}
$$

where $k=\pi$. The formula III.15 is replaced by

$$
\begin{aligned}
u(\xi, \xi) / a= & (1+k)\left[F_{1}(\xi, \xi)-F_{3}(\xi, \xi)\right] \\
& +G_{0}(\xi, \xi)+2 F_{2}(\xi, \xi),
\end{aligned}
$$

where $k=\pi$ and the formula III.18 should be corrected accordingly. The rest of Sec.III. 2 is correct.

\section{L-shaped domain with a horizontal support}

The Sec.III.3 is correct, yet the Table III.1, comprising the results of Sec.III.4 should assume the new form (Table 1).

The volume of an optimal truss is equal to $V=\bar{V} V_{0}$, $V_{0}=\mathrm{Pa} / \sigma_{0}$.

The figure caption of Fig.III.8 should read:

Case of $\theta=\pi / 3, \bar{V}=8.849866$, and $\alpha_{p}=0.162$ to assure $a_{1}=a$.

\section{Optimal topology for special cases}

The figure caption of Fig.III.10 should read:

Case of $\theta=\pi / 2, \bar{V}=16.45864$, and $\alpha_{P}=\pi / 6$.

We repeat here Fig.III.11 as Fig. 1, with small adjustments.

The numerical layout for the problem of Fig. 1 is shown in Fig. 2.

The layout of Fig.III.12 refers only to the case of the force $P$ being vertical. Moreover, the line DN is characterized by $\phi=\theta$ (not $\phi=0$, as printed in Fig.III.12). 
Table 1 Correction of Table III.1. Properties of the special cases in Section III.4

\begin{tabular}{lllllll}
\hline Figure & $\alpha_{P}$ & $\theta$ & $b_{P} / a$ & $a_{1} / a$ & $\bar{V}$ & $\bar{V}_{\text {num }}$ \\
\hline III.7 & 0 & $90 \pi / 180$ & 1 & 1 & 9.283 & 9.28755 \\
& 0.011 & $80 \pi / 180$ & 0.824 & 1 & 9.110106 & 9.11776 \\
& 0.027 & $75 \pi / 180$ & 0.732 & 1 & 9.02813 & 9.03460 \\
& 0.09482 & $65 \pi / 180$ & 0.528 & 1 & 8.89096 & 8.89753 \\
III.8 & 0.162 & $60 \pi / 180$ & 0.408 & 1 & 8.849866 & 8.85448 \\
& 0.323 & $54 \pi / 180$ & 0.222 & 1 & 8.861026 & 8.86247 \\
III.9 & 0.8781 & $50.31 \pi / 180$ & 0 & 1 & 9.007675 & 0.074 \\
III.10 & $\pi / 6$ & $90 \pi / 180$ & 0.788 & 1.792 & 16.45864 & 0.016 \\
III.11 & $\pi / 6$ & $108 \pi / 180$ & 1.358 & 2.008 & 19.1096 & 16.46997 \\
\hline
\end{tabular}

\section{Extended L-shaped domains with an inclined lower boundary}

The formula III.25 holds, where

$V_{1}=\frac{P}{\sigma_{0}} u_{P}\left(\alpha_{P}, \beta_{P}\right)$

and $u_{P}$ is the virtual displacement along the force $P$, as in Fig. 3.

The displacement $u_{P}$ is computed by

$u_{P}=u_{x}\left(\alpha_{P}, \beta_{P}\right) \sin \mu+u_{y}\left(\alpha_{P}, \beta_{P}\right) \cos \mu$

with

$u_{x}(\alpha, \beta)=u(\alpha, \beta) \cos (\beta-\alpha)-v(\alpha, \beta) \sin (\beta-\alpha)$,

$u_{y}(\alpha, \beta)=u(\alpha, \beta) \sin (\beta-\alpha)+v(\alpha, \beta) \cos (\beta-\alpha)$.

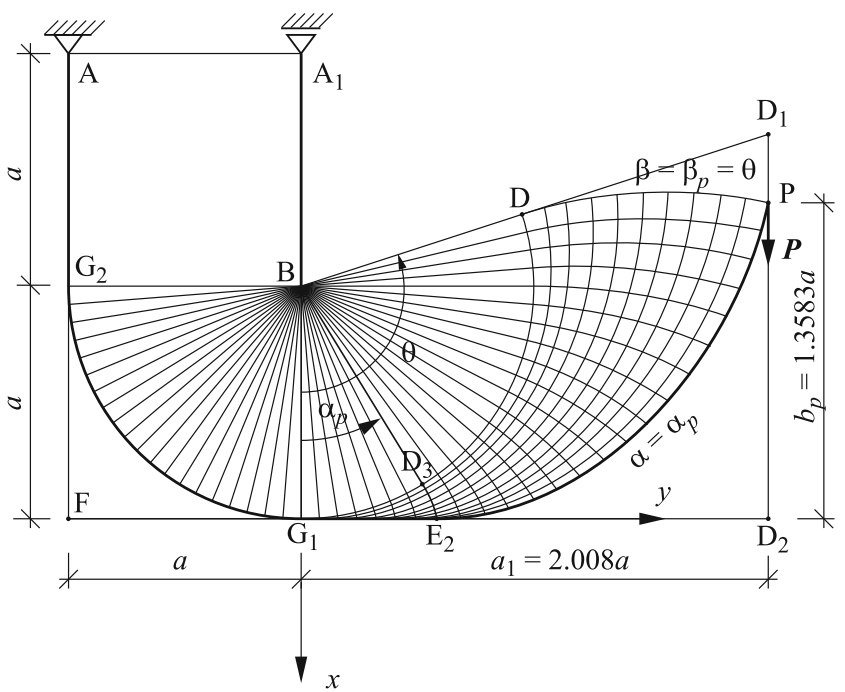

Fig. 1 Case of $\theta=6 \pi / 10, \bar{V}=19.1096$, and $\alpha_{P}=\pi / 6$. The analytical layout
Substitution of (9) into (8) gives

$$
\begin{aligned}
u_{P}(\alpha, \beta)= & u(\alpha, \beta) \sin (\beta-\alpha+\mu) \\
& +v(\alpha, \beta) \cos (\beta-\alpha+\mu)
\end{aligned}
$$

and $u, v$ are given by (5), where one should put $k=2 \mu$. These equations for $u$ and $v$ can be derived from the integral formulae (3), where one should put $\omega_{0}=1+2 \mu$, cf. Eqs.III.24 and II.78.

\section{The cases of the non-vertical point load}

The optimal trusses equilibrating given forces and reactions are composed of two families of bars, one of which in tension and the second in compression. All the nodes, including the supports should be equilibrated. Thus the layout in figure III.12 is valid only for the case of the vertical force-parallel to the bars $\mathrm{AG}_{2}$ and $\mathrm{A}_{1} \mathrm{~B}$. The layouts in

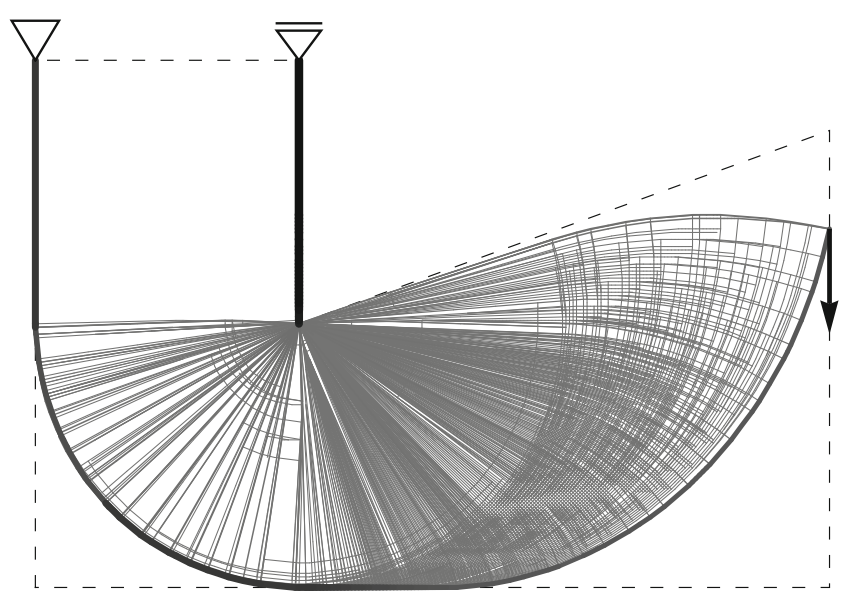

Fig. 2 The numerically determined layout for problem of Fig. 1; the volume of the structure equals $V_{\text {num }}=19.12494 V_{0}$, cf. Table 1 . The result was obtained using the ground structure with $150 \mathrm{mln}$ potential members of 


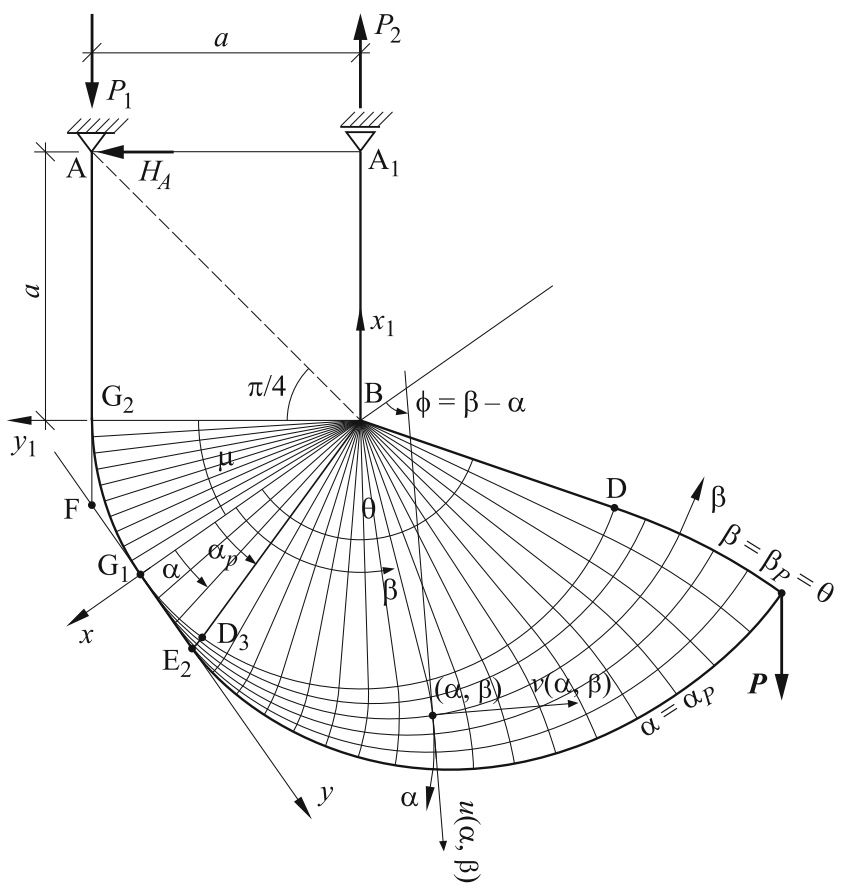

Fig. 3 Case of the inclined lower boundary of the feasible domain; here $\mu=\pi / 5$

Figs III.15, III.16 are incorrect because of the same reason. The numerical solutions to the problems of these figures are shown in Figs. 4 and 5.

The analytical layouts for these two cases (Figs. 4 and 5) will not be constructed, as they lie outside the scope of the layouts discussed here. Instead, let us consider the problem of Fig. 6.

In this problem the reactions $H_{A}=P_{3}$ and $P_{1}$ are positive. Thus the bars which meet at the node $\mathrm{A}$ will have different signs. The analytical layout shown in Fig. 6 will be now explained and parameterized, see Fig. 7. Our main task is to match two virtual displacement fields within the domains: $\mathrm{ABG}_{2}$ and $\mathrm{BG}_{2} \mathrm{D}$ along the radius $\mathrm{BG}_{2}$. Both

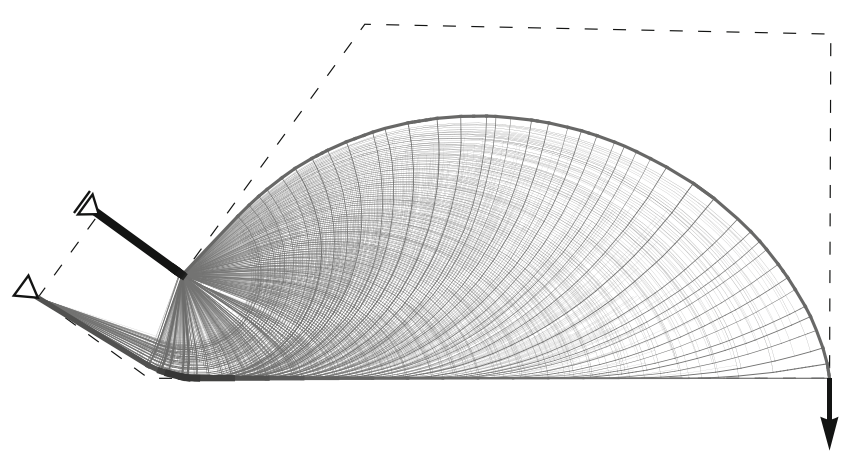

Fig. 4 Numerical solution of the problem equivalent to Fig.III.15, obtained using ground structure with $565 \mathrm{mln}$ of potential members; $V_{\text {num }}=63.63408 V_{0}$

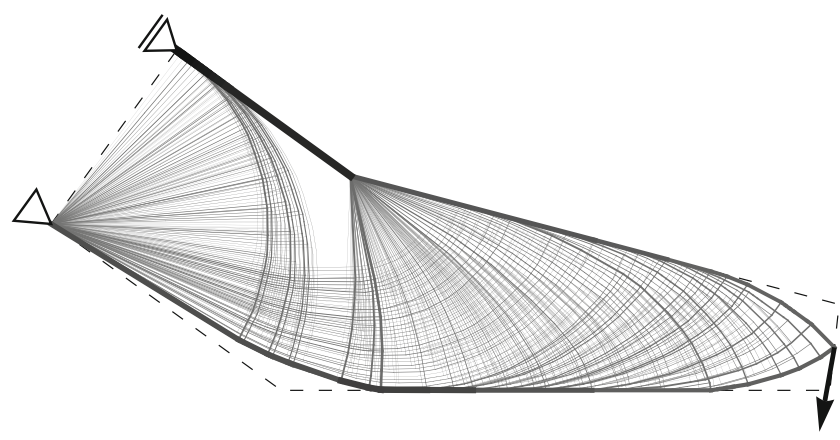

Fig. 5 Numerical solution of the problem equivalent to Fig.III.16, obtained using ground structure with $30 \mathrm{mln}$ of potential members; $V_{\text {num }}=19.34832 V_{0}$

polar parameterizations $\left(r, \beta^{*}\right)$ and $(r, \beta)$ are shown in Fig. 7.

We shall use the formulae (III.2) for both the domains

a) domain $\mathrm{G}_{0} \mathrm{BG}_{2}$ : here $\beta^{*} \in\left(0, \alpha_{0}\right)$, where $\alpha_{0}=50.309^{\circ}$, cf. Fig.III.9

$$
\begin{aligned}
u^{*}\left(r, \beta^{*}\right) & =r, \\
v^{*}\left(r, \beta^{*}\right) & =-2 r \beta^{*}+r, \\
\omega^{*}\left(r, \beta^{*}\right) & =-2 \beta^{*}+1, \omega_{0}^{*}=-1
\end{aligned}
$$

b) domain $\mathrm{BG}_{2} \mathrm{D}: \beta \in(-\mu, \theta)$

$$
\begin{aligned}
& u(r, \beta)=r, v(r, \beta)=-2 r \beta+d_{1} r, \\
& \omega(r, \beta)=-2 \beta+d_{1}, \omega_{0}=-d_{1}
\end{aligned}
$$

The conditions:

$u^{*}\left(r, \beta^{*}\right)=u\left(r,-\beta^{*}-\mu\right) \quad$ and

$v^{*}\left(r, \beta^{*}\right)=-v\left(r,-\beta^{*}-\mu\right)$

assure that the formulae $(11,12)$ describe a continuous displacement field. Note that

$$
\omega^{*}\left(r, \beta^{*}\right)=-\omega\left(r,-\beta^{*}-\mu\right)
$$

because the sign conventions for the rigid rotation are different for both the parameterizations.

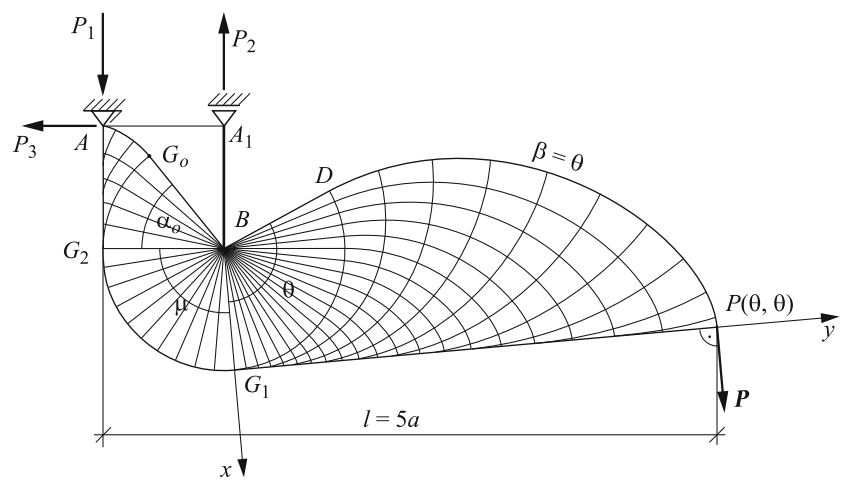

Fig. 6 Problem of class of Fig. 3 for the case of $\mu=95.7106^{\circ}$ and $\theta=113.4456^{\circ}$. The analytically found layout of framework 


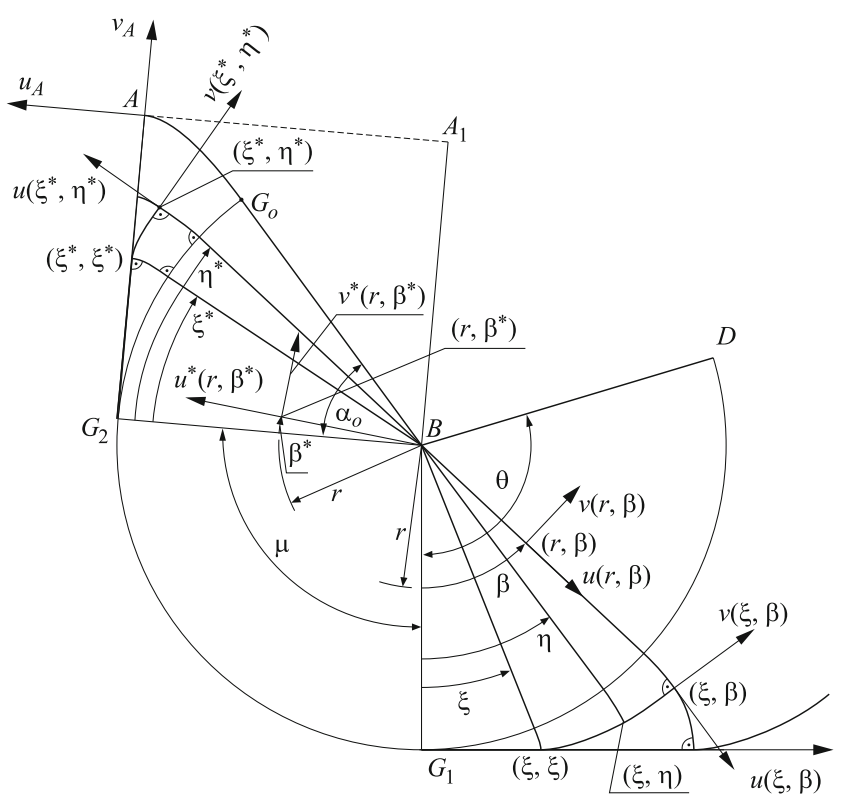

Fig. 7 Polar parameterization around point B

The choice $d_{1}=-1-2 \mu$ will assure that $v_{A}=0$, where $v_{A}$ is denoted in Fig. 7, while $u_{A}$ does not vanish. This choice does not satisfy the kinematic conditions of the problem, but the correct formulae for the optimal weight will be achieved by taking into account the virtual work of the reactions.

Our aim is now to find both virtual displacements $(u, v)$ at arbitrary point $\left(\xi^{*}, \eta^{*}\right)$ of the domain $\mathrm{G}_{0} \mathrm{AG}_{2}$. Let us compute the free terms in (3):

$$
\begin{aligned}
& \bar{u}_{0}\left(\xi^{*}, \eta^{*}\right)=a\left[\cos \xi^{*}-\sin \xi^{*}\left(1-2 \eta^{*}\right)\right] \\
& \bar{v}_{0}\left(\xi^{*}, \eta^{*}\right)=a\left[\sin \xi^{*}+\cos \xi^{*}\left(1-2 \eta^{*}\right)\right]
\end{aligned}
$$

and now compute the displacements: $u\left(\xi^{*}, \eta^{*}\right), v\left(\xi^{*}, \eta^{*}\right)$ by using (3), where we use (15) and put $\omega_{0}^{*}=-1$. Rather lengthy derivation, with using the integration formulae for Lommel functions lead to

$u\left(\xi^{*}, \eta^{*}\right)=a u_{1}\left(\xi^{*}, \eta^{*}\right)$

$$
\begin{aligned}
u_{1}(\xi, \eta)= & 2(\eta-\xi) G_{1}(\xi, \eta)+G_{0}(\xi, \eta) \\
& -F_{1}(\xi, \eta)+2 F_{2}(\xi, \eta)+F_{3}(\xi, \eta),
\end{aligned}
$$

and

$$
\begin{aligned}
v\left(\xi^{*}, \eta^{*}\right)= & a v_{1}\left(\xi^{*}, \eta^{*}\right), \\
v_{1}(\xi, \eta)= & -2(\eta-\xi) G_{0}(\xi, \eta)+G_{0}(\xi, \eta) \\
& -2 F_{1}(\xi, \eta)-2 F_{2}(\xi, \eta)+G_{1}(\xi, \eta),
\end{aligned}
$$

Let us note that

$u_{A}=a u_{1}\left(\alpha_{0}, \alpha_{0}\right), \quad v_{A}=a v_{1}\left(\alpha_{0}, \alpha_{0}\right)$,

where $\alpha_{0}=\angle \mathrm{G}_{0} \mathrm{BG}_{2}=50.309^{\circ}$. Note that $\alpha_{0}$ is the root of the equation

$F_{3}\left(\alpha_{0}, \alpha_{0}\right)-F_{1}\left(\alpha_{0}, \alpha_{0}\right)=-1$.

Note, also that the displacements (16), (18) can be equivalently found from (5) by specifying $k=-2$, because this choice corresponds to a correct value of $\omega_{0}$ for the parameterization in the domain $\mathrm{G}_{1} \mathrm{BPE}_{2} \mathrm{G}_{1}$.

The weight of the optimal structure is given by $V=V_{1}+$ $\Delta V$, where $\sigma_{0} V_{1}$ is equal to the virtual work of the forces $P_{1}, P_{3}, P$ (see Fig. 6) on the virtual displacement field and $\Delta V$ is the weight of the bar $\mathrm{BA}_{1}: \Delta V=\frac{P_{2}}{P} V_{0}$. Thus

$\sigma_{0} V_{1}=P_{3} u_{A}-P_{1} v_{A}+P u(P)$,

where $u_{A}, v_{A}$ are given by (20) and

$u(P)=u(\theta, \theta)$,

where $u$ is defined by (6) for $k=2 \mu$ or

$$
\begin{aligned}
u(P) / a= & (1+2 \mu)\left(F_{1}(\theta, \theta)-F_{3}(\theta, \theta)\right) \\
& +G_{0}(\theta, \theta)+2 F_{2}(\theta, \theta) .
\end{aligned}
$$

Let us note that $v_{A}=0$. Indeed, by (19) we compute

$$
\begin{aligned}
v_{A} / a= & G_{0}\left(\alpha_{0}, \alpha_{0}\right)-2 F_{2}\left(\alpha_{0}, \alpha_{0}\right) \\
& -2 F_{1}\left(\alpha_{0}, \alpha_{0}\right)+G_{1}\left(\alpha_{0}, \alpha_{0}\right) .
\end{aligned}
$$

but $G_{0}=F_{0}+F_{2}, G_{1}=F_{1}+F_{3}$ (cf. (7) in Lewiński et al. 1994) hence

$v_{A} / a=F_{0}\left(\alpha_{0}, \alpha_{0}\right)-F_{2}\left(\alpha_{0}, \alpha_{0}\right)+F_{3}\left(\alpha_{0}, \alpha_{0}\right)-F_{1}\left(\alpha_{0}, \alpha_{0}\right)$.

Recalling the identity (cf. (8) in Lewiński et al. 1994)

$F_{0}(\beta, \alpha)-F_{2}(\beta, \alpha)=\cos (\beta-\alpha)$

and taking into account (21) we conclude that $v_{A}=0$.

The equilibrium equations for the structure of Fig. 6 read

$$
\begin{aligned}
& P_{1}=P[L / a+\cos \mu], \\
& P_{2}=P[L / a+\sin \mu+\cos \mu], \\
& P_{3}=-P \cos \mu,
\end{aligned}
$$


and $L=\left|\mathrm{G}_{1} \mathrm{P}\right|$ or, see Eq.III.26

$L=a\left(F_{1}(\theta, \theta)-F_{3}(\theta, \theta)\right)$.

Let us compute $u_{A} / a$ by (23), (17), (21):

$u_{A}=a\left[G_{0}\left(\alpha_{0}, \alpha_{0}\right)+2 F_{2}\left(\alpha_{0}, \alpha_{0}\right)-1\right]$.

We compute $V$ by (22), (28), (29), taking into account that $v_{A}=0$ :

$$
\begin{aligned}
V / V_{0}= & -\cos \mu\left(u_{A} / a\right)+u(P) / a \\
& +L / a+\sin \mu+\cos \mu .
\end{aligned}
$$

Let us define the function

$$
f(\alpha)=G_{0}(\alpha, \alpha)+2 F_{2}(\alpha, \alpha) .
$$

We rearrange (31) to the form

$$
\begin{aligned}
V / V_{0}= & 2(1+\mu)(L / a)+\sin \mu \\
& +\cos \mu\left[2-f\left(\alpha_{0}\right)\right]+f(\theta) .
\end{aligned}
$$

The above equation is applicable if the net of bars is composed of one family of bars in tension and the second family of bars in compression, mutually orthogonal.

Consider now the problem of Fig. 6 for the data:

$\mu=95.7106^{\circ}, l=5 a$,

where $l$ is the horizontal coordinate of point P, see Fig. 6 . From the equation:

$a(1-\cos \mu)+L \sin \mu=5 a$.

we find $L=3.91995 a$, and by solving (29) we find $\theta=$ $113.4456^{\circ}$. We remember that $\alpha_{0}=50.309^{\circ}$. The volume of the optimal truss is computed by (33), hence $V / V_{0}=$ 42.85857. The analytical layout is shown in Fig. 6, while the numerical layout is given in Fig. 8. The numerically

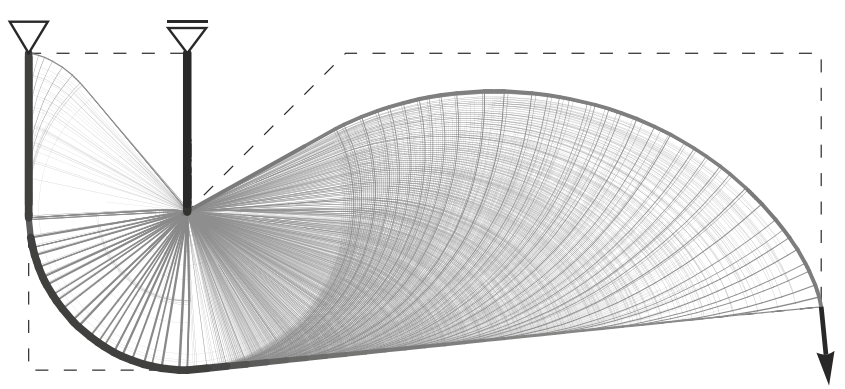

Fig. 8 The numerically found layout of problem of Fig. 6 for the data: $\mu=95.7106^{\circ}, \theta=113.4456^{\circ}$ and ground structure with $474 \mathrm{mln}$ of potential bars; $V_{\text {num }}=42.91234 V_{0}$ found value of the volume equals: $V / V_{0}=42.91234$, which compares favorably with the analytical result.

\section{Final remarks}

The present paper provides new analytical and numerical solutions to the Michell problem, of transmitting a given point load to given supporting nodes, corresponding to the feasible domain being L-shaped, thus being concave, with one reentrant corner. The solutions displayed are characterized by the following features:

- the optimal structures do not make use of the whole feasible domain; some empty domains, around the boundary as well as within the feasible domain appear, see Fig. 5;

- the solutions turn out to be highly sensitive to the load direction, see Fig. 1; the domain between the vertical bars will be (partly) filled up if the load deviates slightly from the vertical direction, see Fig. 6.

All analytical solutions presented in this paper have been confirmed with four-digits precision by the numerical solutions corresponding to highly dense ground structures.

Acknowledgement The paper was prepared within the Research Grant no N506 071338, financed by the Polish Ministry of Science and Higher Education, entitled: Topology Optimization of Engineering Structures. Simultaneous shaping and local material properties determination.

Open Access This article is distributed under the terms of the Creative Commons Attribution License which permits any use, distribution, and reproduction in any medium, provided the original author(s) and the source are credited.

\section{References}

Gilbert M, Tyas A (2003) Layout optimization of large-scale pinjointed frames. Eng Comput 20:1044-1064

Lewiński T, Rozvany GIN (2007) Exact analytical solutions for some popular benchmark problems in topology optimization II: threesided polygonal supports. Struct Multidisc Optim 33:337-349

Lewiński T, Rozvany GIN (2008) Exact analytical solutions for some popular benchmark problems in topology optimization III: L-shaped domains. Struct Multidisc Optim 35:165-174

Lewiński T, Zhou M, Rozvany GIN (1994) Extended exact solutions for least-weight truss layouts — part I: cantilever with a horizontal axis of symmetry. Int J Mech Sci 36:375-398

Sokół T (2011a) A 99 line code for discretized Michell truss optimization written in Mathematica. Struct Multidisc Optim 43:181-190

Sokół T (2011b) Topology optimization of large-scale trusses using ground structure approach with selective subsets of active bars. In: Borkowski A, Lewiński T, Dzierżanowski G (eds) 19th int conf on 'computer methods in mechanics', CMM 2011, 9-12 May, Warsaw, Book of Abstract, pp 457-458 